\title{
Discussion on Fuzzy Synthetical Evaluation for Engineering Construction Quality of Prestressed Concrete Structural
}

\author{
$\mathrm{Liu}^{1}$ Xiaohua \\ Xijing University, Xi'an, 710123, China \\ e-mail: 510924127@qq.com \\ $\mathrm{He}^{3}$ Shidan \\ Xijing University, Xi'an, 710123, China \\ e-mail:315705804@qq.com
}

\author{
$\mathrm{Li}^{2}$ Xiaofang \\ Xijing University, Xi'an, 710123, China \\ e-mail: 563237924@qq.com
}

\begin{abstract}
Objective:This paper discusses the application of fuzzy synthetical evaluating in engineering field especially in building quality of prestressed concrete frame. Methods: The author brings forward mathematic model to fuzzy synthetical evaluating for building quality of prestressed concrete frame which is based on various index integrated evaluating technic and fuzzy mathematics theory. Results: This model synthetically considers all the building indexes which influences the quality of prestressed concrete frame and the correlations of these indexes. And this model evaluates the complex process which is dominated by many indefinitely subjective or objective indexes objectively. Thus qualitative cognitions become to quantitative evaluatings. Conclusion: This evaluating system especially adapts to the evaluation of civil engineering and all kinds of professional engineering 、 large-scale engineering and significant engineering.And This evaluating system has preferably referenced meaning to those ordinary engineerings which have upper qualitatively standard.
\end{abstract}

Keywords-Fuzzy synthetical evaluating Method; prestressed concrete structural ; engineering construction quality

\section{INTRODUCTION}

Construction quality ${ }^{[1]}$ is directly related to the construction engineering quality. To improve construction quality level, we must pay attention to improving the research of construction quality's comprehensive evaluation system .With a scientific measure of construction quality standards, we can establish a sound construction quality control system. Since the construction of a new quality acceptance standards and norms, inspection and assessment carry out"test score off." During the construction phase, the only check on the construction quality is satisfactory. Engineering quality level assessment work is carried out after the completion and acceptance. This evaluation method exists ${ }^{[2]}$ the following problems:

1.Dependent on a handful of experts to judge the quality of the project, the results of such evaluation is often subjective with a strong color.

2.Isolated evaluation of the indicators project, without considering linkages between projects and constraints of each index, the comprehensive evaluation is not in the true sense.

3.Highly professional, high quality requirements, carry out sub-projects complete construction by the professional construction team, according to the current assessment system can not test for quality evaluation, even if it requires the evaluation of a sub-project nor a set of concrete and practical Method.

Based on the views of fuzzy mathematics, This paper proposed a set of "Prestressed Concrete Structures Construction Quality Evaluation System." The system has always been closely linked to the "new standard" and "new norm." From the extracted evaluation factors, to give the evaluation set, and then to get the affiliation of each factor score matrix are "new standard" and "new norms" as the basis. this model evaluates the complex process which is dominated by many indefinitely subjective or objective indexes objectively. Thus qualitative cognitions become to quantitative evaluatings.

\section{FUZZY SyNTHETIC EVALUATION}

Fuzzy Synthetic Evaluation ${ }^{[3]}$ including fuzzy transformation, fuzzy synthetic evaluation of the initial model, multi-level comprehensive evaluation method.

\section{A. Fuzzy transformation}

fuzzy transformation ${ }^{[4]}$ is a very important issue in a comprehensive evaluation. For a limited fuzzy set A can be represented by fuzzy vector, referred to as:

$$
\mathrm{A}=\left(\mathrm{a}_{1}, \mathrm{a}_{2}, \cdots, \mathrm{a}_{\mathrm{n}}\right)
$$

Among, theory of domain:

$$
U=\left(u_{1}, u_{2}, \cdots u_{n}\right)
$$

$\mathrm{a}_{\mathrm{i}}$ is the corresponding membership degree

$$
X_{A}\left(u_{i}\right) i=1,2, \cdots, n
$$


The fuzzy vector can be regarded as a row of columns $\mathrm{n}$ fuzzy matrix, whereby you can define fuzzy transformation.

\section{B. Comprehensive evaluation}

Comprehensive evaluation ${ }^{[5]}$, is for those affected by many factors, or a variety of attributes of things can make a combination of these factors or attributes of the total evaluation. In the case involving the evaluation of fuzzy factors were evaluated using the method of fuzzy results were better.Fuzzy Synthetic Evaluation is the application of fuzzy transformation theory and maximum membership principle, analyzing the various factors associated with the evaluation of things, and do its comprehensive evaluation . Evaluation, necessarily involves a variety of factors. Assumed set of evaluation factors:

$$
U=\left\{u_{1}, u_{2}, \cdots u_{m}\right\}
$$

Often due to the evaluation results of the evaluation work itself requirements, you can use different levels, comments or numerals. Assume that there are $\mathrm{n}$ different rating scale (or comment), they form a collection of evaluation:

$$
V=\left(v_{1}, v_{2}, \cdots v_{n}\right)
$$

First, the factors set $\mathrm{U}$ in the single factor $u_{i}(\mathrm{i}=1,2, \ldots$, $\mathrm{m})$ as the single factor evaluation. From the single factor determining the things $u_{i}$ focus on the evaluation grade $v_{j}(\mathrm{i}=1,2, \ldots, \mathrm{n})$ of membership (possibility degree) $r_{i j}$, so come to the i-th factor $u_{i}$ single factor evaluation set:

$$
r_{i}=\left(r_{i 1}, r_{i 2}, \cdots, r_{i n}\right)
$$

It is a fuzzy set of evaluation set V. Such evaluation set of $\mathrm{m}$ evaluation factors to construct a general evaluation matrix $\mathrm{R}^{[6]}$.

$$
R=\left[\begin{array}{cccc}
r_{11} & r_{12} & \cdots & r_{1 n} \\
r_{21} & r_{22} & \cdots & r_{2 n} \\
\cdots & \cdots & \cdots & \cdots \\
r_{m 1} & r_{m 2} & \cdots & r_{m n}
\end{array}\right]
$$

$\mathrm{R}$ is a fuzzy relation, is to evaluate the factors that domain $\mathrm{U}$ to comment $\mathrm{V}$. $X_{R}\left(u_{i}, v_{j}\right)=r_{i j}$ represents the membership of the evaluation factors $u_{i}$ Level ${ }^{v_{j}}$.In the evaluation, the evaluation of the different factors in the overall evaluation of the degree of influence is different. To this end, various factors need to assign a significant degree ${ }^{u_{i}}$ coefficient $\mathrm{a}_{\mathrm{i}}$. $\mathrm{a}_{\mathrm{i}}$ may be a limiting factor or the adjustment coefficient, it may be common weighting coefficient. The degree of importance of each factor distribution coefficient can be seen as the domain of fuzzy set A on the theory of domain $\mathrm{U}$.

$$
\mathrm{A}=\mathrm{a}_{1} / u_{1}+\mathrm{a}_{2} / u_{2}+\cdots+\mathrm{a}_{m} / u_{m}
$$

Among, $a_{i}(i=1,2, \cdots, m)$ is membership degree from $\mathrm{u}_{\mathrm{i}}$ to A.

C. Multilevel Comprehensive Evaluation Method

When evaluating systems ${ }^{[7]}$ are quite complex, a lot of factors to be considered, which is the existence of two aspects. On the one hand because too many factors, the importance of distribution coefficient is difficult to determine. On the other hand the importance of each factor distribution coefficient is small, like after the adoption of " $\wedge "$ operation a lot of information to be submerged, thereby obtaining no meaningful results. Layered approach to solve these problems.

Factors set sets up:

$$
U=\left\{u_{1}, u_{2}, \cdots u_{m}\right\}
$$

Evaluation set sets up:

$$
V=\left(v_{1}, v_{2}, \cdots v_{n}\right)
$$

Specific steps are as follows ${ }^{[8]}$ :

Factor set is divided into $\mathrm{N}$ subsets by certain properties, namely $U=\left\{u_{1}, u_{2}, \cdots u_{m}\right\}$;

Make $k_{i}$ factors for each of the $U=\left\{u_{1}, u_{2}, \cdots u_{m}\right\}$, according to the initial model, to do comprehensive evaluation;

Assume importance fuzzy set of $U=\left\{u_{1}, u_{2}, \cdots u_{m}\right\}$ is A, so the overall evaluation matrix of $\mathrm{U}$ is R. Among, $A=\left\{A_{1}, A_{2}, \cdots A_{N}\right\}$

$$
R=\left(\begin{array}{c}
B_{1} \\
B_{2} \\
B_{3} \\
B_{4}
\end{array}\right)=\left(\begin{array}{c}
A_{1} \circ R_{1} \\
A_{2} \circ R_{2} \\
\vdots \\
A_{N} \circ R_{N}
\end{array}\right)
$$

So, the secondary comprehensive evaluation result ${ }^{[9]}$ is:

$$
B=A \circ R
$$

This is also the factor $U=\left\{u_{1}, u_{2}, \cdots u_{m}\right\}$ comprehensive evaluation results. If necessary, we can make more of the factors set division level and higher level of comprehensive evaluation. 


\section{FUZZY S YNTHETIC EVALUATION OF PRESTRESSED CONCRETE STRUCTURAL ENGINEERING CONSTRUCTION QUALITY}

\section{A. Create a comprehensive evaluation model}

Based on the introduction of the comprehensive evaluation of fuzzy math and fuzzy basic principles and methods, the purpose of this chapter is to discuss the fuzzy comprehensive evaluation method of prestressed concrete structure engineering construction quality ${ }^{[10]}$

Create a comprehensive evaluation method based on the quality of construction of prestressed concrete structures fuzzy, first establish the factors set comprehensive assessment and evaluation of construction quality set of prestressed concrete structures, follow by the selection comprehensive evaluation model to determine the importance of the factor again, and then determine the membership function, setting scoring standards, establish evaluation matrix, and finally transform the fuzzy comprehensive evaluation results, calculate the comprehensive evaluation score, a comprehensive evaluation.

Selected evaluation factors, both to be able to fully reflect the spirit of construction quality, not too complicated fine principles. In three inspection lot prestressed sub-project - "production and installation," "Tension and release Zhang" and "grout and seal anchor" as the basis of all evaluation factors can be divided into "raw material", "production and installation "," tension "," grout and seal anchor "and" civil engineering with the "five subsets. Each evaluation factors subset and contain several evaluation factors. Such prestressed concrete structural engineering construction quality of evaluation is defined as a two-stage comprehensive evaluation. The classification may determine the quality according to need. You can define evaluation set $\mathrm{V}=$ \{ good, good, fair, poor, very poor\}, you can also define evaluation set $\mathrm{V}=$ \{excellent, good, fair, poor\}, there is no absolute mode.

Evaluation of the factors Determine the importance of the coefficient is one of the most critical aspects of comprehensive evaluation. Currently the most widely used and most effective way is Delphi method, also known as the expert investigation. It is the use of the collective wisdom of experts to determine the evaluation factors in the Comprehensive Assessment of the importance of factors. Experts estimate the qualitative survey are subjective. Different evaluators subjective judgment there will be differences, also due to the subjective judgment of fuzziness affect the clarity of the objective reality. So fuzzy mathematics applied to expert opinion surveys to collect and collate. Experts are not required to come to a consensus, according to the distribution of expert opinion can be calculated importance coefficient of each evaluation factors. Although the importance of Determine the coefficient of using qualitative methods is subjective, but the work was performed prior evaluation of specific projects, thus ensuring the objectivity of the evaluation of specific projects of mass.

\section{B. The actual application of the model}

this paper explore the Nanjing New Century Plaza project to explore fuzzy synthetical evaluating in engineering field especially in building quality of prestressed concrete frame based on Nanjing new century square. Nanjing New Century Plaza project is a podium, and A, B two main body composition. Wherein the B blocks of seven - twenty five (the study of the model) of the frame beam, the beam height is $450 \mathrm{~mm}$, a span is $8.1 \mathrm{~m}$, tendons is $2 \times 5 \Phi \mathrm{s} 15.24$ low relaxation pre-stressed strand, control stress $\sigma=1375 \mathrm{~N} / \mathrm{m} 3$.

Layout Figure is shown as Figure 1. The four side frame girders and two lateral secondary beam are prestressed flat beam.

Fuzzy comprehensive evaluation of specific steps prestressed concrete structural engineering construction quality is as follows:

1. Acceptance calculate scores, and aggregated. The data comes from the construction unit of acceptance records and construction workers collect quality tracking process; 2.Calculation membership, build I level evaluation matrix.

$$
R=\left[\begin{array}{lllll}
R_{1} & R_{1} & R_{1} & R_{1} & R_{1}
\end{array}\right]
$$

3.Through programming, can access to comprehensive evaluation results. 


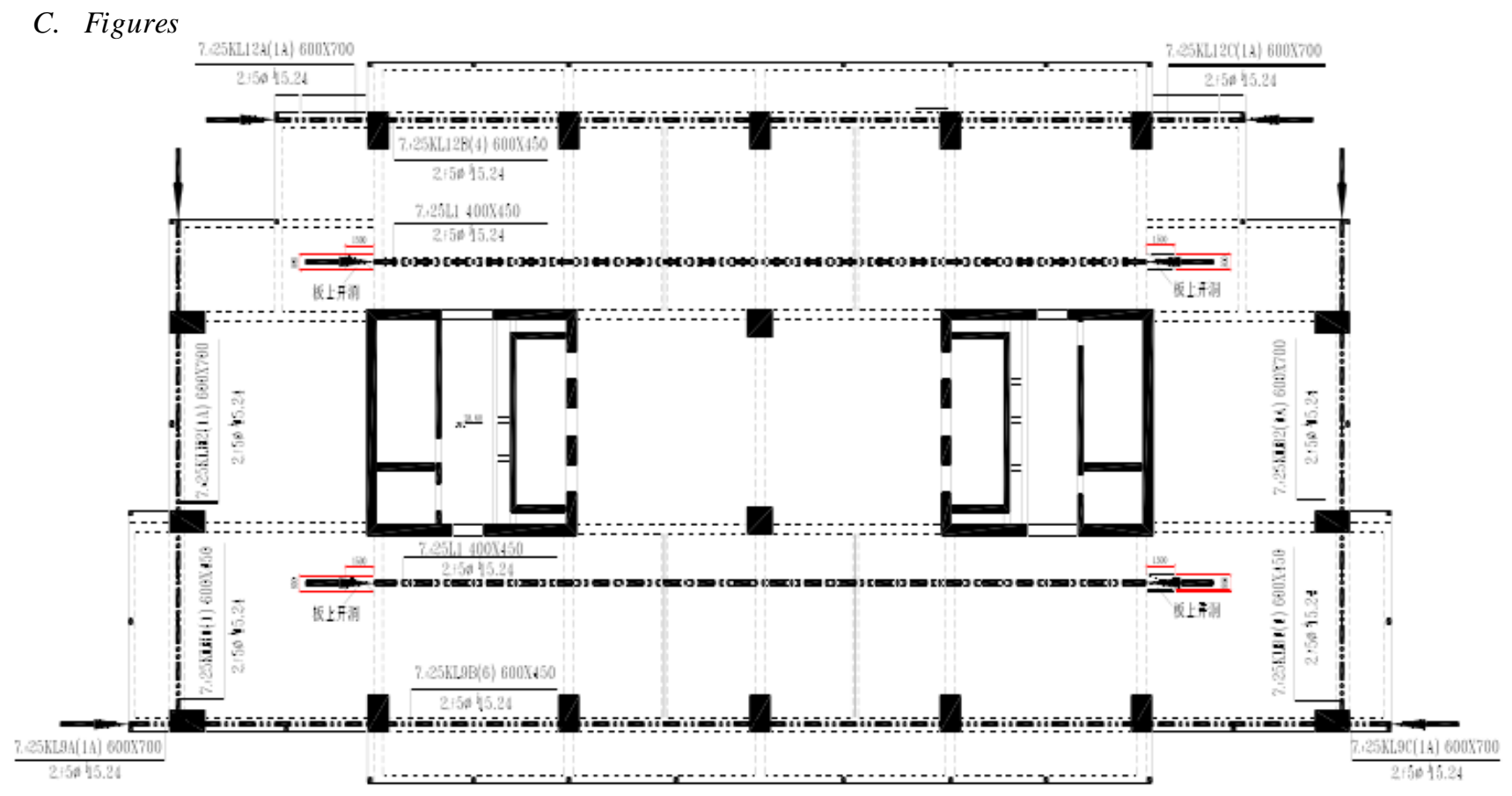

Figure 1. Seven - twenty five prestressed beams plan

\section{CONCLUSIONS}

According to research and analysis over the quality of construction of prestressed concrete structures fuzzy comprehensive evaluation, we reached the following conclusions and recommendations:

1.Evaluation Factors In making any type of project quality evaluation, selection evaluation factors should grasp the "objective and comprehensive, focused" principle; try to select and expand its test items related specifications, procedures or standards requirements, since these projects themselvesIt is predecessor in terms of quality control and quality assessment of lessons learned. Also, note that testing items operability.

2.Membership function In the comprehensive evaluation of multi-index fuzzy, since many factors focus on issues, and strive to find more precise if the membership function of each factor, is unrealistic. But also because the fuzzy comprehensive evaluation, the absolute value of the size of the membership does not make much sense, interest is the comparison between different objects, namely the relative size. As long as the membership function correctly reflect the relative relationship between acceptance points and membership would meet the requirements.

3.Acceptance points The article used acceptance points based on the form of percentile points, unified the various indicators of different data types, so that different types of indicators using the same type of membership function becomes possible.

\section{REFERENCES}

[1] L. B. Wang. "In service remaining bearing capacity of prestressed girder bridge assessment method" D. Chang'an University, 2011.

[2] Q. S. Sun, J. F. Chang. " Highway bridge carrying capacity multilevel fuzzy comprehensive evaluation method "J. Journal of Transportation Systems Engineering and Information Technology. 2008, 01.

[3] P. C. Zhou. "About post-tensioned box girder construction technology " J. Transport World (Construction and Maintenance Mechanical), 2012, 11.

[4] L.A.Zadeh, "Probablility Measures of Fuzzy Events" J. Math Anal App , 1968, 23. Pp. 421-427.

[5] J.F.Baldwin, "A new approach to approximate reasioning using fuzzy logic", Fuzzy Sets and Systems, 1979, 2, pp. 309-325.

[6] G. L. Huang. "Multistage Fuzzy Comprehensive Evaluation Method of prestressed concrete box girder" J. Engineering Economy, 2014,10.

[7] R.R.yager, "Fuzzy Sets,Probabities and Decision", Jour Cybern, 1980. 10. Pp. 1-18.

[8] W. M. Wu, "Fuzzy set theory and its application", Beijing. Beijing Normal University Press, 1985. pp. 35-65.

[9] S. Y. Lu, "Fuzzy synthetical evaluation for building quality of prestressed concrete frame project" D. Southeast University, 2005.

[10] Y. L. Yu. "Experimental study on mechanical properties of corrugated plastic pipe into the hole after prestressed concrete beams" D. School of Civil Engineering, Southeast University, 2003. 